\title{
GAMPI
}

\section{DESENVOLVIMENTO DE PRODUTO - ÓRTESES: REVISÃO SISTEMÁTICA DA LITERATURA.}

Product development - orthotics: systematic literature review.

MATTOZO, Tiago; Pós-Graduado em Marketing Estratégico; Universidade do Vale do Itajaí. tiagomattozo@gmail.com

BATISTA, Vilson João; Pós-Doutor; Universidade Federal de Santa Catarina. vbatista19@gmail.com

\section{Resumo}

O presente artigo descreve a realização de uma revisão de literatura acerca do desenvolvimento de órteses. Para tal, foram conduzidas buscas em duas das mais robustas bases de dados científicos: Scopus e Web of Science. Após a definição das palavras-chave, formação do descritor de busca e adição de filtros, foram selecionados vinte e sete artigos. Destes, oito apresentaram conteúdo relevante ao recorte de pesquisa, enquanto que os outros apenas se aproximaram dos objetivos traçados. Este estudo evidenciou certa carência neste campo de pesquisa, mas, por outro lado, expõe uma área bastante promissora e absolutamente necessária para a qualidade de vida humana.

Palavras Chave: Desenvolvimento de produtos; Órteses; Revisão Sistemática de Literatura.

\section{Abstract}

This article describes a literature review on the development of orthotics. To this end, searches were conducted in two of the most robust scientific databases: Scopus and Web of Science. After defining the keywords, formation of search descriptor and adding filters were selected twenty-seven articles. Of these, eight had relevant content to the research outline, while others only approached the planned objectives. This study showed some lack in this research field, but on the other hand, presents a promising area, absolutely necessary for human life quality.

Keywords: Product development; Orthotics; Systematic Literature Review. 


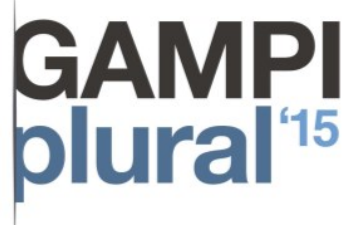

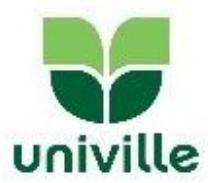

\section{Introdução}

O presente artigo descreve a realização de uma revisão sistemática de literatura, nas bases de dados Scopus e Web of Science, acerca do desenvolvimento de órteses ${ }^{1}$. As buscas nas bases de dados foram realizadas com acesso à rede VPN UFSC, o que possibilitou o alcance a publicações de acesso restrito. As referidas bases de dados foram selecionadas em razão de sua importância acadêmica e o volume de publicações associadas a elas.

O objetivo deste estudo é verificar o estado da arte acerca das produções acadêmicas envolvendo o desenvolvimento de órteses, procurando investigar oportunidades para pesquisas em design. Este estudo também se caracteriza por integrar uma pesquisa mais ampla que visa identificar quais são os requisitos de produto mais relevantes, segundo a percepção dos próprios usuários de órteses.

Este estudo se justifica através de sua contribuição com futuros estudos que se dediquem a abordar este tema - design de produtos, especificamente órteses. A realidade do Brasil, tanto do ponto de vista estatístico quanto tecnológico, evidencia uma preocupante carência de pesquisas científicas e projetos de produtos na área de Tecnologia Assistiva, onde se incluem as órteses.

\section{Procedimentos Metodológicos}

A primeira etapa da revisão foi composta pela seleção das palavras-chave a serem utilizadas nas buscas dentro das bases de dados mencionadas anteriormente. As palavras selecionadas foram utilizadas em dois idiomas: português e inglês. São elas: Tecnologia Assistiva; Assistive Technology; Assistive Device; Órteses; Orthoses; Orthosis. O descritor das buscas então ficou configurado da seguinte maneira: ("Tecnologia Assistiva" OR "Assistive Technology" OR "Assistive Device") AND (Órteses OR Ortheses OR Orthesis). Foram encontrados nas bases Scopus e Web of Science, respectivamente, duzentos e noventa e sete (297) e noventa (90) artigos.

Para que fosse possível atingir uma quantidade de artigos exequível e pertinente à pesquisa, foram aplicados alguns filtros à busca. Foram selecionados então apenas artigos, redigidos no idioma inglês e publicados nos últimos cinco anos (de 2010 a 2014). Com a aplicação dos filtros atingiu-se a marca de sessenta e seis (66) artigos na base Scopus e trinta e três (33) na base Web of Science.

Os noventa e nove (99) artigos encontrados foram então analisados com o apoio do software Zotero ${ }^{2}$ (similar ao Endnote). Após a eliminação das repetições chegou-se a cinquenta e quatro (54) artigos singulares. Mesmo após a aplicação destes diversos filtros, ainda era necessário verificar se os cinquenta e quatro artigos restantes se alinhavam aos objetivos da pesquisa. Foram então lidos e avaliados os títulos, palavras-chave e resumos de

\footnotetext{
${ }^{1}$ Órteses são dispositivos aplicados externamente para modificar as características estruturais e funcionais dos sistemas neuromuscular e esquelético. Fonte: Catálogo Nacional de Produtos de Tecnologia Assistiva. Disponível em <http://www.assistiva.mct.gov.br>. Acesso em: 09 abr. 2014.

2 Zotero é uma ferramenta de pesquisa que recolhe todas as suas pesquisas em uma interface única e pesquisável. Ele permite a adição de PDF's, imagens, arquivos de áudio e vídeo, fotos de páginas da web. O software indexa automaticamente o conteúdo de texto completo de sua biblioteca, permitindo uma busca precisa de conteúdos. Fonte: Wesite Zotero. Disponível em https://www.zotero.org/ Acesso em 09 nov. 2014.
} 


\section{GAMPIII $_{\text {plura }}$}

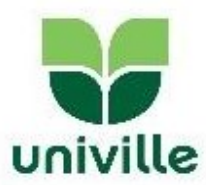

todos os cinquenta e quatro artigos, a fim de eliminar aqueles que se distanciavam dos temas e abordagens propostas.

A partir das destas leituras iniciais, foram eliminados vinte e sete (27) artigos, resultando numa amostra final de, também, vinte e sete (27) artigos. Esses artigos contribuem de alguma forma para o aprofundamento das investigações sobre o desenvolvimento de órteses. Alguns deles se aproximam bastante do tema e abordagem propostos visados, enquanto que outros apenas tangenciam o assunto ou apontam caminhos futuros para que se possa dar continuidade ao presente estudo.

\section{Revisão Sistemática da Literatura}

Dentre os vinte e sete artigos selecionados, oito deles apresentam conteúdo relevante à discussão proposta.

Os estudos desenvolvidos por Magnusson et al (2013) e (2014) são aqueles que mais se aproximam aos objetivos deste estudo, pois investigam a mobilidade e satisfação dos usuário de próteses e órteses de membros inferiores. Basicamente, os dois estudos apresentam as mesmas características, diferenciando-se apenas quanto ao recorte geográfico. Enquanto que a pesquisa de 2013 foi desenvolvida com usuários Malauianos ${ }^{3}$, a de 2014 foi realizada em Serra Leoa.

No estudo de Magnusson et al (2013), os usuários Malauianos informaram que priorizavam o acesso à manutenção e os reparos, seguido pela durabilidade e serviços de acompanhamento. Já a pesquisa de Magnusson et al (2014), realizada em Serra Leoa, foi identificado que $33 \%$ dos usuários apresentavam quadro de dor durante o uso de seu dispositivo assistivo e muitos apresentavam dificuldade de mobilidade em terreno irregulares $(65 \%)$, ladeiras $(75 \%)$ ou escadas $(66 \%)$. Ainda assim, os usuários estavam bastante satisfeitos com o seu dispositivo assistivo e os serviços associados a ele. Os resultados também identificaram oitocentos e oitenta e seis (886) problemas. O estudo apontou que, dentre todos os usuários, aqueles que utilizam dispositivos assistivo acima do joelho apresentaram os piores resultados.

Plos et al (2012) apresentam um modelo de referência exclusivamente voltado ao desenvolvimento de produtos de TA, chamado EMFASIS. A sigla do nome do modelo significa Extended Modularity, Functional Accessibility and Social Integration Strategy (Estratégia de Integração Social, Acessibilidade Funcional e Modularidade Estendida). Como forma de validar o modelo proposto, os pesquisadores apresentam três produtos desenvolvidos segundo as premissas do EMFASIS. Um destes produtos é uma órtese para membros superiores.

Farris, Quintero e Goldfarb (2011) também desenvolvem uma avaliação de órtese de membro inferior. Contudo, focam-se em um dispositivo motorizado analisando sua contribuição para a marcha de um indivíduo paraplégico. Esta revisão pretendia abordar apenas órteses mais simples e comuns, fabricadas em material termoplástico (polipropileno), ou seja, aquelas que não se utilizam de propulsão mecânica ou elétrica. Esse recorte se deve a

\footnotetext{
${ }^{3}$ Relativo ou pertencente à República do Malauí, leste da África. sm O natural ou habitante dessa República. Sin: malaviano. Fonte: Dicionário Michaelis. Disponível em: http://michaelis.uol.com.br/moderno/portugues/index.php?lingua=portugues-portugues\&palavra=malauiano Acesso em: 10 jan. 2015.
} 


\section{GAMPI
plural ${ }^{15}$}

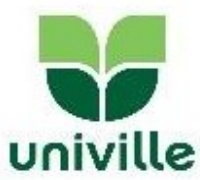

maior profusão e menor custo deste tipo de órtese, o que a torna mais acessível às pessoas com deficiência, que, em sua maioria, são indivíduos com poucos recursos financeiros.

No estudo realizado por Çakar et al (2010) investigou-se a contribuição da órtese tornozelo-pé para o equilíbrio e redução do risco de queda em usuários acometidos por hemiparesia espástica crônica ${ }^{4}$. Os autores da pesquisa trabalharam com uma amostra de vinte cinco (25) indivíduos e identificaram resultados positivos em relação às melhorias supracitadas. Direta, ou indiretamente, essas melhorias oferecem uma melhor qualidade de vida a seus usuários. Os autores frisam que os resultados mais significativos ocorreram em indivíduos que apresentavam espasticidade moderada após a ocorrência do Acidente Vascular Cerebral (AVC). Em casos que apresentam espasticidade mais acentuada, as órteses contribuem positivamente, mas também se exige a presença aproximada de um cuidador, dado que o equilíbrio do indivíduo esta muito mais comprometido. Sendo assim, nesses casos, a órtese contribui, mas apresenta um papel secundário no equilíbrio do indivíduo, pois o principal agente de melhoria concentra-se na figura do cuidador.

Fujak et al (2011) realizaram uma pesquisa extensa, utilizando cento e noventa e quatro (194) indivíduos, com o intuito de determinar o uso de dispositivos assistivos (cadeiras de rodas, coletes e órteses ) a pacientes com atrofia muscular espinal ${ }^{5}$. Os resultados desta pesquisa enfatizam a importância de melhorar as ações de fornecimento de órteses e dispositivos assistivos, assim como incrementar os processos de adaptação às necessidades individuais do paciente.

Andreoni et al (2014) têm como objetivo a formulação de um protocolo de medição para apoiar decisões clínicas. Os autores consideram que, mesmo a partir de uma pequena amostragem (4 indivíduos), os resultados indicam um futuro promissor para o método testado, mas que ainda precisa ser trabalhado e testado novamente. Mesmo sendo direcionado às avaliações clínicas, este estudo, assim como outros, também ressalta a importância do processo de desenvolvimento e customização de uma órtese para a qualidade de vida do usuário.

Em seu estudo, Gupta et al (2010), investigam a reabilitação e os déficits residuais advindo da Síndrome de Guillain-Barre ${ }^{6}$ (SGB). Sua investigação resulta na identificação das órteses para membros inferiores mais adequadas aos indivíduos acometidos por esta síndrome. A partir de uma amostra composta por trinta e cinco (35) indivíduos, os autores identificaram que vinte e um usuários apresentavam pé-caído ${ }^{7}$, necessitando de órtese de

\footnotetext{
${ }^{4}$ Hemiparesia espástica crônica é um tipo de paralisia cerebral. O paciente apresenta movimentos normais do lado sadio e restrição da movimentação do lado afetado, que se torna normalmente ignorado. (PALÁCIO; FERDINANDE; GNOATTO, 2008, p. 127).

${ }^{5}$ A atrofia muscular espinhal é uma doença neurodegenerativa progressiva. O paciente acometido pela AME necessita de vários cuidados especiais, que podem estacionar o progresso da doença e prolongar a vida do mesmo. (BAIONE; AMBIEL, 2010, p. 262).

${ }^{6}$ Síndrome de Guillain-Barré é uma polineuropatia aguda de rápida progressão que causa debilidade simétrica progressiva e ascendente dos membros. (BENETI; SILVA, 2006, p. 58).

${ }^{7}$ Pé caído é uma disfunção nervosa periférica que causa paralisia do músculo tibial anterior. (SILVA et al, 2006, p. 473).
} 


\section{gAMPIII $^{\text {GAMP }}$}

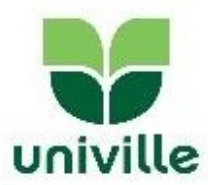

tornozelo-pé. Além disso, trinta dos trinta e cinco pacientes necessitavam de dispositivo assistivos para sua locomoção. Segundo os pesquisadores, os pacientes com SGB têm déficits residuais, mesmo utilizando órtese, mas mostram uma significativa recuperação funcional, ou seja, há melhoria em suas capacidades de realizar as atividades da vida diária.

Apesar de fazerem parte da seleção final da pesquisa sistemática, os outros dezenove artigos não apresentam fortes elementos de comunhão com os objetivos desta revisão. Sua inclusão justifica-se pela exemplificação dos tipos de estudos que têm sido desenvolvidos sobre órteses.

O recorte de pesquisa mais comum entre esses artigos é a análise da marcha a partir do uso de algum tipo de órtese. Uchiyama, Nagai e Obinata (2012), Bae, Kong e Tomizuka (2012), Lugrís et al (2013), Andrysek, Klejman e Kooy (2014), Chin, Hsiao-Wecksler e Loth (2012), McCain, Smith e Querry (2012) e Yoon, Kumar e Ozer (2012) são os autores encontrados que relacionam exclusivamente a marcha ao uso de órtese, sejam elas comuns ou propulsionadas. Evidentemente a marcha, ou o caminhar, é fundamental para a acessibilidade e inclusão social das pessoas com deficiência, no entanto, o objetivo desta revisão é investigar os aspectos que contribuam com o desenvolvimento de órteses, tal como a satisfação geral dos usuários sobre as órteses.

Os dois artigos publicados por Hwang et al (2012) e (2013) também analisam o resultado nos parâmetros da marcha de indivíduos com AVC a partir do uso de órteses.

Farris e Sawicki (2012), Shorter et al (2011), Farris et al (2014), Shamaei, Sawicki e Dollar (2013), Owens et al (2011) e Sartori et al (2012) apresentam pesquisas onde o objeto de estudo são órteses propulsionadas ou exoesqueletos, ou seja, diferem do tipo de órtese aqui em questão.

No estudo conduzido por Kirchner et al (2014) buscou-se a detecção dos movimentos intencionais dos pacientes a partir de dados de eletroencefalograma ou eletromiografia. Seus objetivos, no entanto, focavam-se muito mais no progresso terapêutico da reabilitação e não no desenvolvimento de recursos de TA.

Trazendo uma abordagem singular, Colombo et al (2013) estudam os custos envolvido no tratamento da doença Espinhal Bífida. Como resultado, os pesquisadores identificaram que os maiores custos estão envolvidos com os produtos de Tecnologia Assistiva necessários ao tratamento. Esse artigo alerta para uma questão socialmente relevante, em razão da baixa capacidade econômica da maioria das pessoas com deficiência.

Zupan e Jenko (2012) apresentam um levantamento sobre as opções de recursos de TA para pessoas com paralisia cerebral. Dentre as opções os autores citam e descrevem dispositivos de locomoção (modelos de cadeiras de rodas) e dispositivos de comunicação aumentativa e alternativa.

\section{Discussão}

Quase que instantaneamente, esta revisão de literatura expõe um cenário ainda incipiente acerca da produção científica sobre o desenvolvimento de produtos de Tecnologia Assistiva, tais como órteses. Em um país como o Brasil, que conta com mais de quarenta milhões de pessoas com deficiência (segundo dados do CENSO 2010), fica evidente um campo de estudo promissor e ainda pouco explorado, especialmente para designers envolvidos com pesquisas científicas. Por se tratar de uma área do conhecimento que, por essência, visa compreender e contribuir com os anseios das pessoas 


\section{pAMPI II $^{\text {GA }}$}

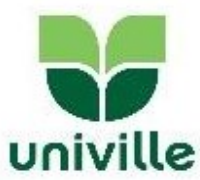

(usuários/clientes/consumidores), o design certamente possui seu espaço nesse processo evolutivo.

Sobre os resultados obtidos com a presente revisão de literatura, fica claro que os primeiros oito artigos descritos apresentam uma maior consonância com os objetivos traçados, pois realmente abordam aspectos relacionados ao desenvolvimento de produtos, focando-se em órteses. Em geral, abordam o desenvolvimento de órteses, apontam evoluções no processo de reabilitação e, consequentemente, na qualidade de vida de seus usuários. Portanto, pesquisas que pretendem aprofundar esse assunto e alargar as fronteiras acerca destes conhecimentos precisam estar atentas às contribuições descritas nestes oito artigos. Os dezenove artigos seguintes, tangenciam o recorte de pesquisa proposto por esta revisão de literatura e, ainda que de forma pontual, oferecem contribuições para o design de produtos de Tecnologia Assistiva.

Em ambos os estudos desenvolvidos por Magnusson et al (2013 e 2014) concluiu-se que os usuários estavam satisfeitos com sua prótese/órtese e com os serviços associados a elas. Contudo, ainda assim, inúmeros problemas foram relatados pelos usuários ou identificados pelos pesquisadores, problemas estes que indicam demandas passíveis de serem contempladas por soluções advindas do design.

A contribuição do design também fica evidente no estudo conduzido por Plos et al (2012), pois, visando à execução de protótipos, formou-se um grupo de trabalho composto por diversos indivíduos, inclusive pessoas com deficiência e designers. Este estudo representa mais uma produção científica envolvendo o desenvolvimento de TA que valoriza a participação dos usuários no processo de concepção e planejamento do produto, assim como preveem os métodos de design.

Os resultados encontrados por Fujak et al (2011) ajudam a justificar a relevância do assunto aqui abordado, bem como da realização desta revisão de literatura, visto que reforçam a necessidade de melhorar as soluções para as reais necessidades dos usuários. Neste mesmo sentido, Andreoni et al (2014) afirmam que "a escolha correta e customização de uma órtese são cruciais para obter o melhor conforto e eficiência." (p. 1). O aprofundamento em questões relacionadas com o design de produtos sempre tem como premissa a facilitação das atividades humanas e a melhoria da qualidade de vida. Neste sentido, esta revisão mostra que o caminho a ser percorrido ainda é longo, mas também amplo e pouco explorado por designers.

\section{Conclusão}

Com relação aos procedimentos adotados, é possível concluir que, para a realização de uma revisão de literatura relevante, no mínimo dois aspectos são fundamentais: domínio do idioma inglês e adoção de corretos filtros de busca. A importância do primeiro aspecto se manifesta no fato de que o volume de conhecimento científico é imensamente maior nos países Europeus e, principalmente, nos Estados Unidos. Portanto, é imprescindível que os pesquisadores científicos tenham um bom domínio do idioma inglês. O segundo aspecto relevante, correta adoção de filtros, se justifica, pois, com os avanços tecnológicos da internet, o acesso à informação se tornou vasto. Com isso, a adoção de filtros de busca é absolutamente necessária e precisa ser executada de forma a produzir os conteúdos mais atuais e relevantes para as pesquisas que se pretende realizar.

Sobre os resultados obtidos, pode-se concluir que o design, não somente enquanto área de pesquisa, mas também como ramo de atividade, deve atentar-se às demandas associadas à 


\section{GAMPI
plural ${ }^{15}$}

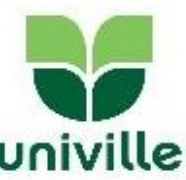

Tecnologia Assistiva. Somente no Brasil, mais de quarenta e cinco milhões de pessoas (usuários/clientes/consumidores) urgem por melhores produtos e serviços mais eficientes. Seja pelos aspectos funcionais, ou mesmo estético-simbólicos, o design certamente tem capacidade de promover melhorias reais à qualidade de vida deste vasto grupo de pessoas.

\section{Referências}

ANDREONI, Giuseppe et al. Wearable monitoring devices for assistive technology: Case studies in Post-Polio Syndrome. Sensors, [s. L.], v. 14, n. 2, p.2012-2027, jan. 2014. Disponível em: $\quad<\mathrm{http} / /$ www.scopus.com/inward/record.url?eid=2-s2.084893020396\&partnerID $=40 \& m d 5=e 8037$ eae6b861a381a63f748488debca $>$. Acesso em: 25 ago. 2014.

ANDRYSEK, Jan; KLEJMAN, Susan; KOOY, John. Forces and moments in kneeankle-foot orthoses while walking on irregular surfaces: A case series study. Prosthetics And Orthotics International, Toronto, v. 38, n. 2, p.104-113, abr. 2014. Disponível em: $<\mathrm{http}: / / \mathrm{www}$.scopus.com/record/display.url?eid=2-s2.084902191270\&origin=inward\&txGid=EE0F2B2402C74B3E912916DE9ECD195E.aqHV0Eo E4xlIF3hgVWgA:388>. Acesso em: 25 ago. 2014.

BAE, Joonbum; KONG, Kyoungchul; TOMIZUKA, Masayoshi. Gait phase-based control for a rotary series elastic actuator assisting the knee joint. Journal Of Medical Devices: Transactions of the ASME, [s. L.], v. 5, n. 3, p.1-6, ago. 2011. Disponível em: $<\mathrm{http}$ //www.scopus.com/inward/record.url?eid=2-s2.0-

80051863623\&partnerID $=40 \& m d 5=$ cd72c6179e94494b72fde8c14b0eae24>. Acesso em: 25 ago. 2014.

BAIONI, Mariana T. C.; AMBIEL, Celia R.. Spinal muscular atrophy: diagnosis, treatment and future prospects. Jornal de Pediatria. Porto Alegre, p. 261-270. jul. 2010. Disponível em: <http://www.scielo.br/pdf/jped/v86n4/a04v86n4.pdf>. Acesso em: 12 fev. 2015.

BENETI, Giselle Maria; SILVA, Dani Luce Doro da. Síndrome de Guillain-Barré. Semina: Ciências Biológicas e Saúde, Londrina, v. 27, n. 1, p.57-69, jan. 2006. Disponível em: <http://www.uel.br/revistas/uel/index.php/seminabio/article/view/3529/2856>. Acesso em: 10 fev. 2015.

BOBATH, Karel. Uma base neurofisiológica para o tratamento de paralisia cerebral. 2. ed. São Paulo: Manole, 1984.

ÇAKAR, Engin et al. The ankle-foot orthosis improves balance and reduces fall risk of chronic spastic hemiparetic patients. European Journal Of Physical And Rehabilitation Medicine, [s. L.], v. 46, n. 3, p.363-368, set. 2010. Disponível em: $<$ http://www.scopus.com/inward/record.url?eid=2-s2.0-

79251610300\&partnerID $=40 \& m d 5=$ fa2f4da1088504e $869863 \mathrm{~d} 7753 \mathrm{de} 8 \mathrm{~d} 2 \mathrm{~b}>$. Acesso em: 25 ago. 2014. 


\section{GAMPI
plural ${ }^{45}$}

CHIN, Robin; HSIAO-WECKSLER, Elizabeth; LOTH, Eric. Fluid-power harvesting by under-foot bellows during human gait. Journal Of Fluids Engineering, Transactions Of The Asme, [s. L.], v. 134, n. 8, p.1-7, jul. 2012. Disponível em: $<$ http://www.scopus.com/inward/record.url?eid=2-s2.084864571536\&partnerID $=40 \&$ md5 $=3$ feab18a716e30575703ec4cf2cc439a $>$. Acesso em: 25 ago. 2014.

COLOMBO, Giorgio L. et al. A cost-of-illness study of spina bifida in Italy. Clinicoeconomics And Outcomes Research, Pavia, v. 5, n. 1, p.309-316, jul. 2013. Disponível em: $<$ http://www.scopus.com/inward/record.url?eid=2-s2.084879723828\&partnerID $=40 \& m d 5=10 \mathrm{~b} 0 \mathrm{a} 15 \mathrm{c} 0 \mathrm{a} 08 \mathrm{f} 35 \mathrm{a} 2 \mathrm{f} 52326150580 \mathrm{fe} 8>$. Acesso em: 25 ago. 2014.

FARRIS, Dominic James; SAWICKI, Gregory S.. Linking the mechanics and energetics of hopping with elastic ankle exoskeletons. Journal Of Applied Physiology, [s. L.], v. 113, n. 12, p.1862-1872, dez. 2012. Disponível em: $<$ http://www.scopus.com/inward/record.url?eid=2-s2.0-

84871558494\&partnerID $=40 \& \mathrm{md} 5=\mathrm{b} 05 \mathrm{e} 2833 \mathrm{a} 703 \mathrm{f} 6 \mathrm{fle}$ e $\mathrm{ec} 0 \mathrm{c} 4 \mathrm{c} 386 \mathrm{cafc} 5>$. Acesso em: 25 ago. 2014.

FARRIS, Ryan J.; QUINTERO, Hugo A.; GOLDFARB, Michael. Preliminary evaluation of a powered lower limb orthosis to aid walking in paraplegic individuals. IEEE Transactions On Neural Systems And Rehabilitation Engineering, Nova Iorque, v. 19, n. 6, p.652-659, dez. 2011. Disponível em: $<$ http://www.scopus.com/inward/record.url?eid=2s2.0-83455220178\&partnerID=40\&md5=6e16ddf77e90ae421 ebad0d8aa7dc44b $>$. Acesso em: 25 ago. 2014.

FARRIS, Ryan J et al. A preliminary assessment of legged mobility provided by a lower limb exoskeleton for persons with paraplegia. IEEE Transactions On Neural Systems And Rehabilitation Engineering, [s. L.], v. 22, n. 3, p.482-490, maio 2014. Disponível em: $\quad<$ http://www.scopus.com/inward/record.url?eid=2-s2.084900444768\&partnerID $=40 \& m d 5=0 \mathrm{c} 99 \mathrm{~d} 71 \mathrm{e} 1 \mathrm{c} 4 \mathrm{c} 180 \mathrm{ccc} 9 \mathrm{fa} 3 \mathrm{~b} 2 \mathrm{~d} 355 \mathrm{ed} 16>$. Acesso em: 25 ago. 2014.

FUJAK, Albert et al. Use of orthoses and orthopaedic technical devices in proximal spinal muscular atrophy: Results of survey in 194 SMA patients. Disability And Rehabilitation: Assistive Technology, [s. L.], v. 6, n. 4, p.305-311, jul. 2011. Disponível em: $<\mathrm{http}$ ://www.scopus.com/inward/record.url?eid=2-s2.0-

79955821713\&partnerID=40\&md5=e1 f95fc90226664a6bdbb59914d5ad3a $>$. Acesso em: 25 ago. 2014.

GUPTA, Anupam Datta et al. Guillain-Barre Syndrome: Rehabilitation outcome, residual deficits and requirement of lower limb orthosis for locomotion at 1 year followup. Disability And Rehabilitation, [s. L.], v. 32, n. 23, p.1897-1902, jan. 2010. Disponível 


\section{GAMPI
plural ${ }^{15}$}

em:

$<$ http://www.scopus.com/inward/record.url?eid=2-s2.0-

77957781406\&partnerID $=40 \& m d 5=654 \mathrm{bb} 2 \mathrm{~d} 8 \mathrm{dc} 0 \mathrm{bf} 2 \mathrm{e} 337 \mathrm{a} 58373 \mathrm{fa} 1 \mathrm{e} 3 \mathrm{eca}>$. Acesso em: 25 ago. 2014.

HWANG, Youngin; An, Dukhyun; YOO, Wongyu. Effects of the Dual AFO on gait parameters in stroke patients. Neurorehabilitation, [s. L.], v. 31, n. 4, p.387-393, jan. 2012. Disponível em: $\quad<$ http://www.scopus.com/inward/record.url?eid=2-s2.0$84872393145 \&$ partnerID $=40 \& \mathrm{md} 5=068172 \mathrm{e} 5 \mathrm{~d} 1801 \mathrm{df} 8 \mathrm{f} 82 \mathrm{~d} 1848 \mathrm{aa} 416 \mathrm{a} 34>$. Acesso em: 25 ago. 2014.

HWANG, Youngin; YOO, Wongyu; AN, Dukhyun. Effects of the Elastic Walking Band on gait in stroke patients. Neurorehabilitation, [s. L.], v. 32, n. 2, p.37-322, jan. 2013. Disponível em: $\quad<\mathrm{http} / /$ www.scopus.com/inward/record.url?eid=2-s2.0$84876221960 \&$ partnerID $=40 \& m d 5=24 c c 58 b 03$ fa585bac4a9b729b0eab873 $>$. Acesso em: 25 ago. 2014.

KIRCHNER, Elsa Andrea; TABIE, Marc; SEELAND, Anett. Multimodal movement prediction: Towards an individual assistance of patients. Plos One, [s. L.], v. 9, n. 1, p.110, jan. 2014. Disponível em: $<$ http://www.scopus.com/inward/record.url?eid=2-s2.084893822751\&partnerID $=40 \& m d 5=062$ ebaee8efee $02525 \mathrm{e} 26 \mathrm{ce} 1$ eafc $4 \mathrm{f} 6 \mathrm{c}>$. Acesso em: 25 ago. 2014.

LUGRÍS, Urbano et al. Gait analysis system for spinal cord-injured subjects assisted by active orthoses and crutches. Proceedings Of The Institution Of Mechanical Engineers: Part K: Journal of Multi-body Dynamics, [s. L.], v. 227, n. 4, p.363-374, dez. 2013. Disponível em: <http://www.scopus.com/inward/record.url?eid=2-s2.084896762009\&partnerID $=40 \& m d 5=0 \mathrm{e} 46 b b b d 6672484 \mathrm{e} 617015 \mathrm{~d} 996086988>$. Acesso em: 25 ago. 2014.

MCCAIN, Karen J.; SMITH, Patricia Sherwood; QUERRY, Ross G.. Ankle-Foot orthosis selection to facilitate gait recovery in adults after stroke: A case series. Journal Of Prosthetics And Orthotics, Dallas, v. 24, n. 3, p.111-121, jul. 2012. Disponível em: $<$ http://www.scopus.com/inward/record.url?eid=2-s2.0-

84864275577\&partnerID=40\&md5=b193f56827999876590d275e8f594b87>. Acesso em: 25 ago. 2014.

MAGNUSSON, Lina et al. Malawian prosthetic and orthotic users' mobility and satisfaction with their lower limb assistive device. Journal Of Rehabilitation Medicine, [s. L.], v. 45, n. 4, p.385-391, fev. 2013. Disponível em: $<$ http://www.scopus.com/inward/record.url?eid=2-s2.0-

$84876000541 \&$ partnerID $=40 \& m d 5=b 93 a 5 f 0 c 882 b 5 d d 70 e e 3 a 68 f 53015525>$. Acesso em: 25 ago. 2014.

MAGNUSSON, Lina et al. Mobility and satisfaction with lower-limb prostheses and orthoses among users in Siera Leone: A cross-sectional study. Journal Of Rehabilitation 


\section{GAMPI
plural 15}

Medicine, [s. L.], v. 46, n. 5, p.438-446, mar. 2014. Disponível em: $<$ http://www.scopus.com/inward/record.url?eid=2-s2.0-

84902984683\&partnerID=40\&md5=2519c32ba7c78c676ab99f340f1cb73b $>$. Acesso em: 25 ago. 2014.

OWENS, Johnny G. et al. Return to running and sports participation after limb salvage. Journal Of Trauma: Injury, Infection and Critical Care, [s. L.], v. 71, n. 1, p.120-124, jul. 2011. Disponível em: <http://www.scopus.com/inward/record.url?eid=2-s2.079960385441\&partnerID=40\&md5=2f1387a680840aa968b232e8c8ad4939>. Acesso em: 25 ago. 2014.

PALÁCIO, Siméia Gaspar; FERDINANDE, Ariadne Katia Soares; GNOATTO, Francielle Cristina. Análise do desempenho motor de uma criança com hemiparesia espástica pré e pós-tratamento fisioterapêutico: estudo de caso. Ciência, Cuidado e Saúde, Maringá, v. 7, n. 1, p.127-131, dez. 2008. Disponível em: $<$ http://periodicos.uem.br/ojs/index.php/CiencCuidSaude/article/view/6583/3896>. Acesso em: 20 fev. 2015.

PLOS, Ornella et al. A Universalist strategy for the design of Assistive Technology. International Journal Of Industrial Ergonomics, Amsterdam, v. 42, n. 6, p.533-541, 28 set. 2012. Disponível em: <http://www-sciencedirectcom.ez46.periodicos.capes.gov.br/science/article/pii/S0169814112000881\#>. Acesso em: 02 abr. 2014.

SARTORI, Massimo et al. Modeling the human knee for assistive technologies. Ieee Transactions On Biomedical Engineering, [s. L.], v. 59, n. 9, p.2642-2649, jul. 2012. Disponível em: $\quad<\mathrm{http} / / /$ www.scopus.com/inward/record.url?eid=2-s2.084865449753\&partnerID $=40 \& m d 5=9 \mathrm{c} 65 \mathrm{e} 655 \mathrm{c} 238 \mathrm{ac} 6411 \mathrm{a} 1817 \mathrm{eee} 4813 \mathrm{ed}>$. Acesso em: 25 ago. 2014.

SHAMAEI, Kamran; SAWICKI, Gregory S.; DOLLAR, Aaron M.. Estimation of Quasi-Stiffness and Propulsive Work of the Human Ankle in the Stance Phase of Walking. Plos One, [s. L.], v. 8, n. 3, p.1-10, mar. 2013. Disponível em: $<$ http://www.scopus.com/inward/record.url?eid=2-s2.0-

84875317694\&partnerID $=40 \& m d 5=829916486 \mathrm{dc} 467 \mathrm{eb} 1 \mathrm{~b} 260 \mathrm{~b} 9 \mathrm{~b} 8 \mathrm{daec} 509>$. Acesso em: 25 ago. 2014.

SHORTER, Kenneth Alex et al. A portable powered ankle-foot orthosis for rehabilitation. Journal Of Rehabilitation Research And Development, [washington], v. 48, n. 4, p.459-472, jan. 2011. Disponível em: $<$ http://www.scopus.com/inward/record.url?eid=2s2.0-79957862497\&partnerID=40\&md5=71a94f7dfbb3f49b72e8>. Acesso em: 25 ago. 2014.

SILVA, Julio Guilherme et al. Coerência espectral do eletrencefalograma em pacientes submetidos a transposição tendinosa: estudo pré e pós-operatório. Arquivos de 


\section{GAMPIII $_{\text {plura }}$}

Neuropsiquiatria, São Paulo, v. 64, n. 2, p.473-477, jun. 2006. Disponível em: $<$ http://www.scielo.br/pdf/anp/v64n2b/a23v642b.pdf>. Acesso em: 18 fev. 2015.

UCHIYAMA, Yoshiho; NAGAI, Chikara; OBINATA, Goro. Simulation of human walking with powered orthosis for designing practical assistive device. Conference Proceedings: Annual International Conference of the IEEE Engineering in Medicine and Biology Society, San Diego, v. 1, n. 1, p.4816-4819, ago. 2012. Disponível em: $<$ http://www.scopus.com/inward/record.url?eid=2-s2.0-

84903864809\&partnerID=40\&md5=2d0cc0292a1b68addb69f7263b3b4a8a $>$. Acesso em: 25 ago. 2014.

WEISZFLOG, Walter (Ed.). Dicionário Michaelis. [São Paulo]: Melhoramentos, 2012. Disponível em: <http://michaelis.uol.com.br/moderno/portugues/index.php>. Acesso em: 20 jan. 2015.

YOON, Jungwon; KUMAR, R. Prasanth; ÖZER, Abdullah. An adaptive foot device for increased gait and postural stability in lower limb orthoses and exoskeletons. International Journal Of Control, Automation And Systems, [s. L.], v. 9, n. 3, p.515-524, jun. 2011. Disponível em: <http://www.scopus.com/inward/record.url?eid=2-s2.0-

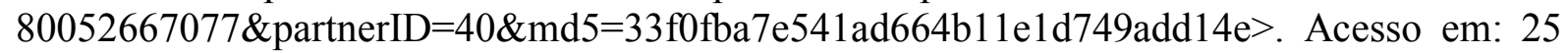
ago. 2014.

ZUPAN, Anton; JENKO, Mojca. Assistive technology for people with cerebral palsy. Eastern Journal Of Medicine, van, v. 17, n. 4, p.194-197, out. 2012. Disponível em: $<$ http://www.scopus.com/inward/record.url?eid=2-s2.0-

84875715582\&partnerID $=40 \& m d 5=7 \mathrm{a} 133 \mathrm{bf} 2823584$ ee72f7b34b0c2db019>. Acesso em: 25 ago. 2014. 